\title{
Orion II: The Second Generation Readout Multiplexer for the Largest Infrared Hybrid Focal Plane
}

\author{
K. M. Merrill, A. Fowler, and W. Ball, NOAO \\ A. Henden and F. Vrba, USNO Flagstaff \\ C. McCreight, NASA Ames
}

\begin{abstract}
The Orion program developed a 2048x2048 infrared focal plane using InSb PV diodes for detectors. Several of these focal planes have been produced. However, the yield of the original readout multiplexer was not up to expectations owing to unanticipated shorts in the fabrication process. Since these shorts occurred at the metal 1-metal 2 crossover points and there are over 9 million such crossovers, the design had to be modified to work around these problems. Thus the Orion II readout was developed. The work is being done at the Raytheon Vision Systems (RVS) division (most recently Raytheon Infrared Operations, but better known as SBRC) by many of the same people who created the Orion I and ALADDIN focal planes. The design is very similar to the Orion I design with the addition of circuitry to work around the effect of the metal 1-metal 2 shorts. In this paper we will discuss the unique design features of this device as well as present test data taken from the new devices.
\end{abstract}

Keywords: Large-format arrays, Infra-Red (IR), Focal Plane Array (FPA), InSb, astronomical detectors, IR multiplexers

\section{INTRODUCTION}

The Orion program - to develop on behalf of the astronomical community a $2048 \times 2048$ infrared focal plane for groundbased astronomy using InSb PV diodes for detectors - is in its third year. The Orion program was officially started in the spring of 2001, but informal discussion of the design started much earlier. In late CY2000, with funding support from NOAO, USNO, and NASA, we laid out a program to develop a $2 \mathrm{Kx} 2 \mathrm{~K}$ InSb focal plane at RVS similar to the successful ALADDIN 1Kx1K program. We had our first bare readout/multiplexer for test in late 2001 and to date have made five focal plane modules. Four of those modules are complete hybrids. Testing has proceeded at both NOAO and RVS on those modules. Orion program goals, design specifics, and early results have been reported elsewhere ${ }^{1,2}$. The readout design is solid but, as will be spelled out in detail below, some changes were deemed to be necessary to improve yield.

The Orion design has several features that are either new or relatively recent additions to IR focal planes. It is specifically designed with 2 -adjacent side butt-ability in mind. In addition, we have adopted a modular packaging scheme that facilitates the deployment of tightly integrated $4 \mathrm{Kx} 4 \mathrm{~K}$ focal plane assemblies that is reflected in the James Webb Space Telescope (JWST) version of this sensor ${ }^{3}$, where 3 -side buttability is the norm. Based on the aggregate cost of the instruments, electronics, and focal planes, we felt that $4 \mathrm{Kx} 4 \mathrm{~K}$ was a prudent design goal at this time. Indeed, such a 4 Orion module mosaic is slated for the NEWFIRM imager at $\mathrm{NOAO}^{4}$ currently scheduled for deployment in CY2005. The demonstrated success of using dead pixels for calibration purposes ${ }^{5}$ led us to incorporate several different reference pixel ideas into this design. Recent testing has shown their usefulness in removing the effects of thermal drift from the data $^{2}$. Other improvements are that the focal plane is read out as a single $2 \mathrm{Kx} 2 \mathrm{~K}$ device rather than in quadrants and that it reads out in stripes of 32 columns per output rather than interleaved as has been done in the past with multiple output devices. Bad outputs are manifested as a contiguous dead area rather than missing periodic columns across the whole device. Finally, with 64 outputs, the Orion design is designed to meet the conflicting needs of ground-based astronomy to perform well and with high efficiency in low, moderate, and high background environments.

We stayed with the reliable, proven 2 micron, Pwell, CMOS, Single Polysilicon, Double Metal process for Orion. With the exception of double metal, this is the same process we used on Aladdin ${ }^{6}$. Double metal was needed because of the long lines $(>50 \mathrm{~mm})$ in both directions across the readout. This did affect the yield but was required to keep the line impedance low or the speed of readout would have been unacceptable. Since Orion "only, has 64 outputs, we needed to maintain the $1.5 \mu \mathrm{sec}$ per pixel $(5 \tau)$ read time or its usage would have been severely compromised operation in the thermal infrared. 


\section{DESIGN}

\subsection{Specification}

Specifications and goals for the Orion device are summarized below in Table 1.

\begin{tabular}{|c|c|}
\hline \multicolumn{2}{|r|}{ Table 1: Orion Program Specifications and Goals } \\
\hline Format & $2048 \times 2048$ \\
\hline Pixel Size & $25 \times 25$ microns \\
\hline Array Active Area & $51.2 \times 51.2 \mathrm{~mm}$ \\
\hline Optical Fill Factor & $>98 \%$ \\
\hline Architecture & $\begin{array}{l}\text { Two Side Buttable (Adjacent sides) } \\
\text { Designed to make a } 4 \mathrm{~K} \times 4 \mathrm{~K} \text { larger module } \\
64 \text { outputs: read out in stripes of } 32 \text { columns each } \\
\text { Built-in current source per output } \\
\text { Read out as a single } 2048 \times 2048 \text { device } \\
\end{array}$ \\
\hline Readout Structure & $\begin{array}{l}\text { Source Follower per Detector (SFD) Unit Cell } \\
\text { Pwell CMOS Control Logic } \\
\text { On Chip Buffers for all clocks and control signals } \\
\text { Control Logic-2 phase shift registers } \\
\end{array}$ \\
\hline References & $\begin{array}{l}\text { First (1) and last columns (2048) are reference columns } \\
\text { Additional references selectable on each output as follows: } \\
\text { 1) reference, } 32 \text { data pixels, reference } \\
\text { 2) reference, } 32 \text { data pixels } \\
\text { 3) } 32 \text { data pixels only }\end{array}$ \\
\hline Reset Modes & \begin{tabular}{|l|} 
Global (all detectors) \\
Ripple (Reset by row pairs)
\end{tabular} \\
\hline Output Performance & * $1.5 \mu$ sec per pixel $(5 \tau)-($ Frame rate $\sim 10$ frames per second $)$ \\
\hline Detector Material & Backside-illuminated - thinned p-on-n InSb \\
\hline Well Capacity & $150 \times 10^{3}$ electrons at $0.5 \mathrm{v}$ applied bias \\
\hline Read Noise & $<25$ electrons RMS for Fowler 1 sampling \\
\hline Dark Current & $<0.2$ electrons per second per pixel (for $\mathrm{T}<=35 \mathrm{~K}$ ) \\
\hline Quantum Efficiency & $>95 \%$ at 1.7 microns (Quarter wave AR coating at 1.7 microns) \\
\hline Response & 0.6 to 5 microns with appropriate AR coating \\
\hline Operating Temperature & 25-35 Kelvin \\
\hline Operability & Typically $>99.5 \%$ \\
\hline
\end{tabular}

\subsection{Packaging Concept}

The packaging concept used on the Orion program is a major departure from previous IR array packaging approaches. We needed a concept that would package the $2 \mathrm{Kx} 2 \mathrm{~K}$ Orion focal plane and also readily accommodate expansion to $4 \mathrm{Kx} 4 \mathrm{~K}$ mosaics. The important features of the Orion Focal Plane Module (FPM) are identified in Figure 1. These include the use of 37 pin MDM connectors (which we have used in the past for cryogenic applications and we know are reliable) on ribbon cables to provide positive connectivity while providing thermal isolation and the use of an AlN motherboard for the optimal placement of the electrical traces while providing electrical isolation from the Invar36 pedestal. The electrical isolation is important, as the pedestal can be hard clamped to the cold head while the Sensor Chip Assembly (SCA) detector itself, of necessity, remains electrically isolated for noise reasons. Both the Invar36 and AlN where chosen for their thermal compatibility with the silicon readout. In addition the invar pedestal was made over $6 \mathrm{~mm}$ thick to provide mechanical stiffness and thermal mass. This has proven successful since we have had no cracks or delamination of indium bumps with over $30 \mathrm{cool}$ downs. To mitigate the effects of light emission from current sources or from the area where the cables are mated to the motherboard, we have included $2.5 \times 0.6 \mathrm{~mm}$ AlN light baffles to separate 
the detector SCA from stray emission sources. The under side of the pedestal has several threaded holes for mounting the module to a larger detector mount. In addition there is one hole located in the optical center of the detector for maintaining alignment with respect to an instrument if needed. For $4 \mathrm{Kx} 4 \mathrm{~K}$ modules there are provisions for pins and corresponding holes in the side of the pedestal for alignment of multiple $2 \mathrm{~K} \times 2 \mathrm{~K}$ sub-modules.

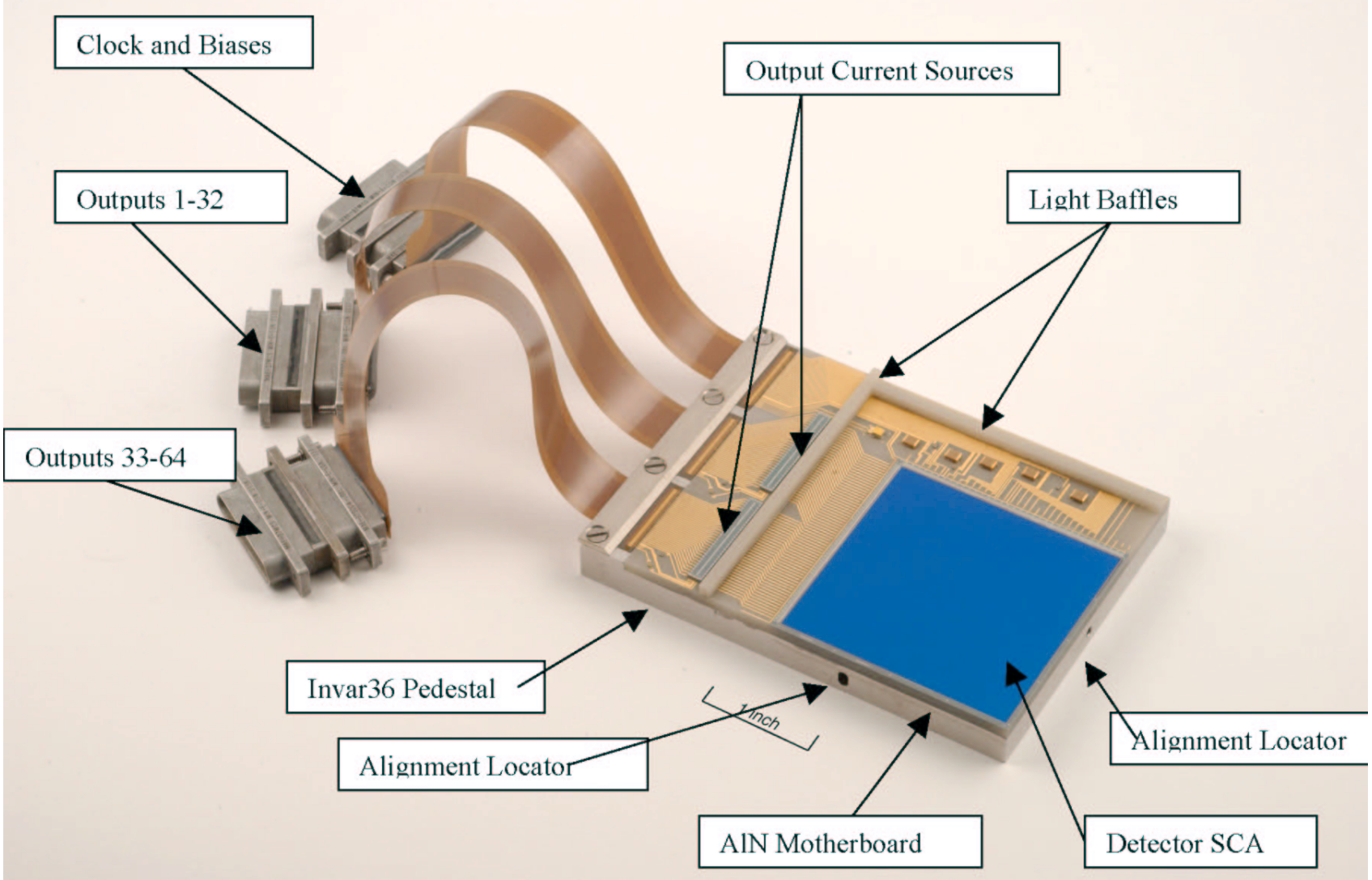

Figure 1. Orion 2Kx2K Focal Plane Module

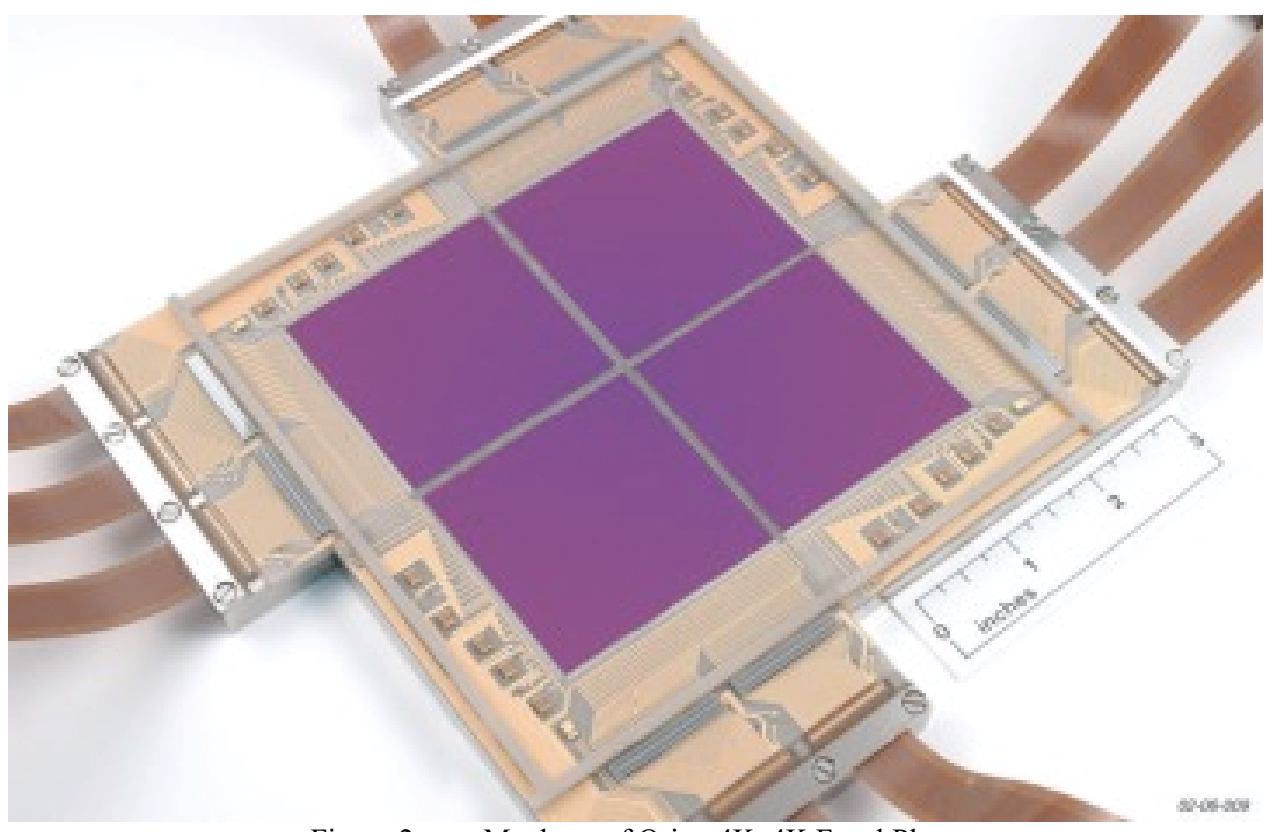

Figure 2. $\quad$ Mock-up of Orion 4Kx4K Focal Plane 
As you can see in Figure 2, these baffles completely surround the active areas in a $4 \mathrm{Kx} 4 \mathrm{~K}$ implementation. The modules are designed to directly fit snugly, reliably, and safely together into a 2 by 2 mosaic. Many of these concepts are shared with the 3 side buttable 2Kx2K FPA InSb modules developed by RVS for the JWST mission ${ }^{3}$. The synergy between the Orion and JWST development efforts is summarized elsewhere ${ }^{7}$. It is worth noting that both these efforts underway at RVS are being carried out by many of the same people who created the ALADDIN focal planes.

\subsection{Internal References}

The Orion design has several internal references to aid in noise reduction, removal of thermal affects, and uniformity correction. The first and most important of these references is the Output Reference, which is a unit cell multiplexed directly into the output bus by the fast shift register (state $\mathrm{C} 0$ ). The gate of this unit cell is tied directly to VdetCom, which is the reference level for all detector measurements. Drifts in this bias translate directly into changes in the measured output signal. By appropriate clocking, it can be inserted into the data stream at the beginning as well as the end of each row read. In this way, it could be used to remove the thermal drift in the output unit cell and the output stage as well as $1 / \mathrm{f}$ noise from the beginning to the end of the row read. Figure 3 shows an extreme example where the output stage drift was successfully removed using this Output Reference capability.

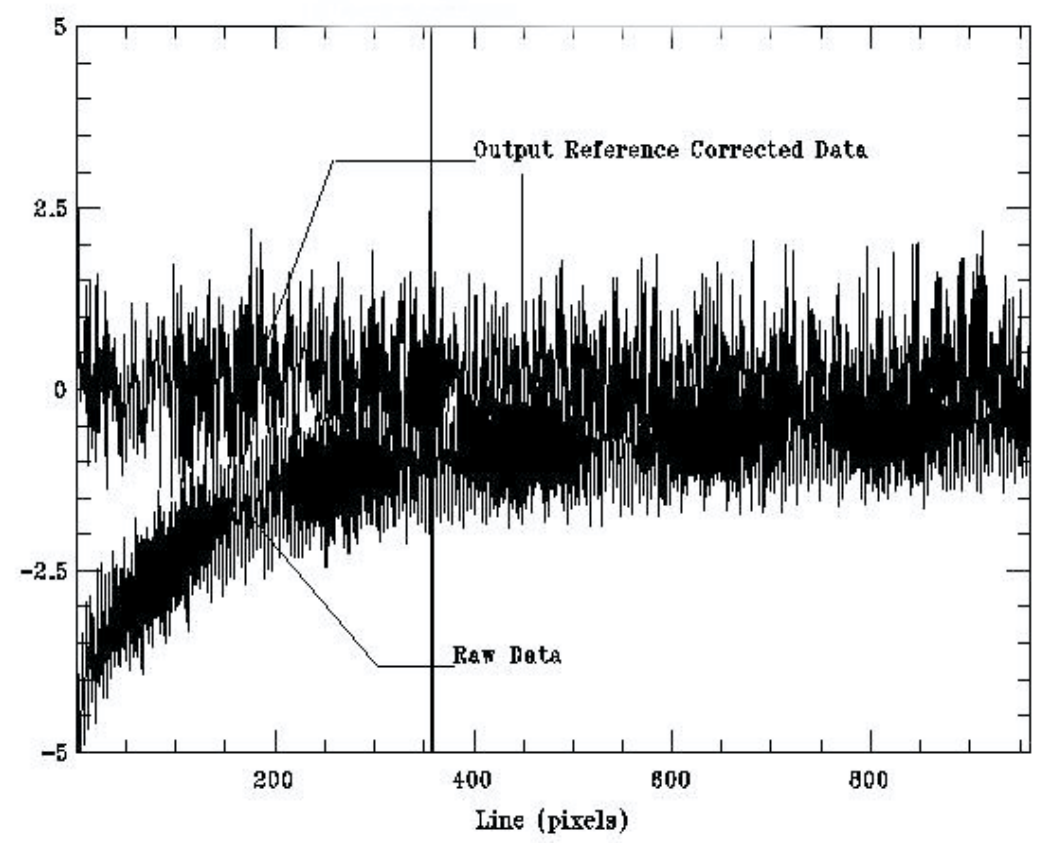

Figure 3 Data Signal Improvement Using the Output Reference (signal ordinate is in ADUs)

The second set of references are data columns 1 and 2048. The column 1 unit cells are connected to capacitors, whose value is similar to the detectors, and are reset like the detectors. They could be used for row to row uniformity correction. In column 2048 there are no reset transistors and the unit cells are connected to VdetCom like the Output References. Hence reference data are available at both the dark and saturation levels for possible uniformity correction. Over time we intend to experimentally determine which of these references are the most useful.

\subsection{Current Sources}

Output current sources for the Orion device, produced on the readout wafer along with the multiplexers, have been mounted directly on the focal plane module (see Fig. 1). They can be powered on or off through a single externally available line. Detection of glow from the output amplifiers has been the bane of IR focal planes and numerous and 
variegate attempts have been made to mitigate such effects. We reasoned that by turning the outputs completely off, we could limit the deleterious effect of parasitic radiation from amplifier glow on long integrations. This is of particular concern to the Orion design, because we have increased the physical size of the output transistors to improve their capacity to drive a load.

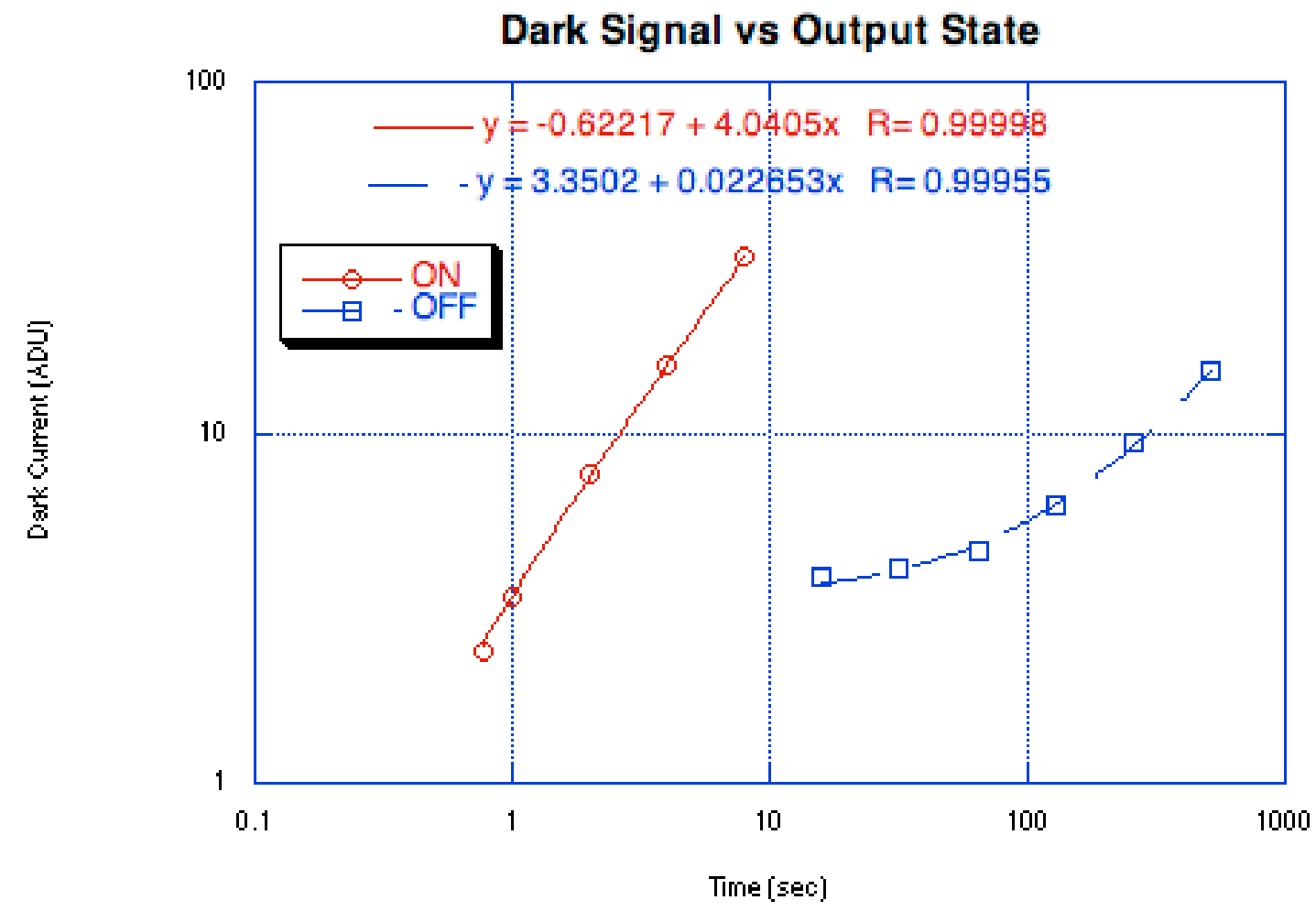

Figure 4. Efficacy of controlling the power to the on-chip detector outputs: Left curve (circles) have the power on throughout the integration. Right curve (squares) have the power off when not being read.

Figure 4 illustrates the positive impact of this feature on dark signal. When measuring darks, we normally take a sequence of 10 exposures starting at the minimum integration time and extending from 1 to 512 seconds in powers of 2 employing Fowler_1 sampling. In the sequence shown, we were leaving the outputs turned on for integrations less than 10 seconds and powering them off between reads for integrations longer than 10 seconds. Although the cause of the relatively high rates with the outputs left on is not yet fully understood, turning the current sources off obviously had a dramatic effect on the measured background.

\section{THE NEED FOR THE ORION II REDESIGN}

\subsection{The Challenge}

The Orion design is a 2-micron, single poly-silicon, double metal process. The departure from the double polysilicon, single metal design successfully employed by the ALADDIN arrays was done to reduce the RC time constant in the reset and row_enable lines. Otherwise, the increased resistance in the polysilicon lines - owing to the factor of two increase in overall linear dimension and the reorganization of the basic chip architecture from a four quadrant to a monolithic design (an aggregate increase of a factor of 4) - would have seriously curtailed operation of Orion devices at high background. 
The Orion readout design is fundamentally solid, meeting or surpassing our goals in terms of read noise, settling time, and general operability. However, initial testing of the first lot of Orion readouts uncovered a serious problem inherent to the design. Since there are over 9 million metal crossovers, the aggregate probability of defects due to metal to metal shorts using the 2-micron process is non-negligible. Possible short conditions include:

1. the row_enable line to the unit cell Vdduc ( $>2$ million crossings)

2 . the row_enable line to the output bus ( $>4$ million crossings)

3. the reset_enable line to Vdduc ( $>1$ million crossings)

4. the reset_enable line to the output bus (>2 million crossings)

The first possibility led to in a very serious problem: if a row_enable line shorted to the unit cell Vdduc (the unit cell drain voltage) level, it would result in that row being enabled all the time. Therefore, with two or more rows (the addressed one and the shorted one) being enabled, the signal from the addressed row is compromised. The reality of this deleterious effect has been confirmed in both theory (with PSpice modeling) and in practice (using a laser point source). The output of the multi-connected regions that is nearer the reset value (where the wells are emptier) dominates the answer - in essence, dark rules. Since the extent of the effect depends on the signal levels of the pixels in the row with the short and the correctly addressed row, it follows that de-convolving the result under typical astronomical observing conditions at moderate to low background would be nearly impossible. Since this scenario accounts for over 2 million crossovers, the possibility of making a multiplexer without any Vdduc to row_enable shorts is problematic. Indeed, there appeared to be no devices in Lot 1 that were free of the defect. The other short conditions listed do not result in the loss of the whole array due to multiple-connection confusion. They might result in a lost row or column but with 4 million pixels that amounts to only $0.05 \%$ (an insignificant amount).

\subsection{The Solution}

Based on the results of Lot 1 , which led to discovery of the problem, with no devices free of the "shorted row_enable to Vdduc,, defect, a design change was clearly required for Orion. After reviewing the layout carefully, we concluded that there was nothing in the layout itself that contributed to this problem; hence the Orion redesign - Orion II. Early in the ALADDIN $1 \mathrm{Kx} 1 \mathrm{~K}$ program we determined the need for a way to disable a row because of shorts between the long polysilicon lines across the die and incorporated this feature into the design. By introducing a row_pair reset approach in later ALADDIN implementations, we eliminated this particular problem, but still retained the capability to disable a row if required. We had incorporated this same design feature into the original Orion design, but it only applied to rows and not to columns (since columns had not shown to be a problem with the ALADDIN design). In the Orion II design we extended the row disable feature to the columns as well.

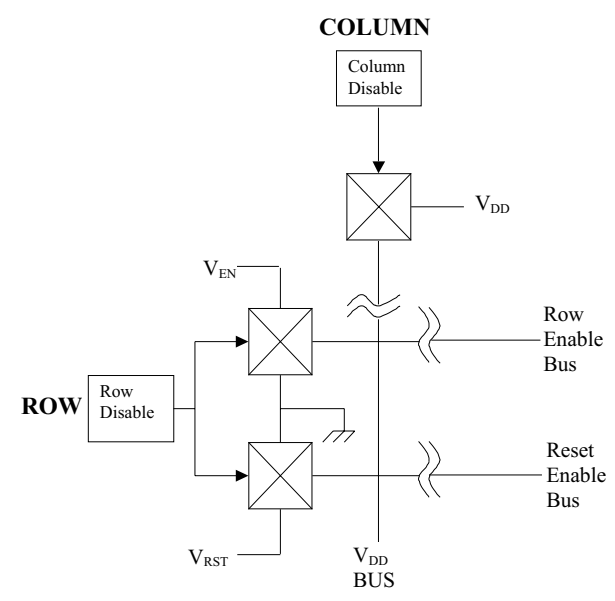

Figure 5. Row and column disable logic block diagram for the Orion II readout

The disable design (see the block diagram in Figure 5) contains a flip-flop that is addressed by the shift register and set with the disable clock. For the rows the output of the flip-flop controls a switch that grounds the selected row and reset enables for the selected row pair. In the case of the columns the output of the flip-flop controls a FET switch and it opens 
the Vdduc line to the column pair. In this manner, the Vdduc column line with the short can be disabled and the affected row enable line can be grounded by the row_disable logic. Although you lose two columns and possibly two rows for each shorted location, that is a small price to pay for recovering the rest of the die. Depending on the severity of the short it may not be necessary to disable the affected row.

Shorts to the output bus will result in a loss of data from the effected column but do not affect the rest of the readout. Shorts from the Vdduc line to the reset line have little or no effect, as resetting requires a level of over $1.5 \mathrm{v}$ more negative to operate the reset transistor. Shorts from the reset line to the output bus likewise have no effect beyond the affected column.

\subsection{Yield results}

We have completed the initial Lot of Orion 2 readouts (Lot 2, 24 wafers, 72 readouts), tested the disable feature, and have verified that it works as planned. We have taken devices exhibiting the "shorted row_enable to Vdduc,, defect and recovered them using the row and column disable circuitry. As shown in Table 2, this has improved the yield from nearly zero to over $50 \%$ devices with better than $99.5 \%$ good pixels. These results are conservative, since the actual defects may be effect fewer rows and columns than seems indicated. It is also worth noting that, based on wafer probe tests, several devices from Lot 2 appear to be defect free.

\begin{tabular}{|c|c|}
\hline \multicolumn{2}{|c|}{ Table 2 } \\
\hline Orion 2 yield employing row/column disable \\
\hline Operability & Yield \\
\hline$>=99.5 \%$ & $50 \%$ \\
\hline$>=99.8 \%$ & $28 \%$ \\
\hline
\end{tabular}

\section{TESTING}

\subsection{Status of the NOAO Test System}

Testing at NOAO has been limited by the capabilities of the present test system. A new system, based on the Monsoon system currently under development at $\mathrm{NOAO}^{8}$ has been delayed and is not expected to be available until later this year. Meanwhile, we have modified the venerable Wildfire based NOAO Infrared Research and Development Lab array controller ${ }^{9,}{ }^{10}$, used to test the ALADDIN arrays ${ }^{11}$, and pressed it into service to establish a solid basis for comparing the performance of the ALADDIN and Orion devices. Although this hybrid array controller has only 8 channels, it is sufficiently flexible to meet our near-term test needs and has the distinct advantage of familiarity when it comes to rapid development and deployment of new software-codes while the ultimately more capable Monsoon system is under active development. The new test dewar ${ }^{2}$, which was designed to test the Orion FPM's as well as future 4Kx4K IR mosaics is working well. It can be pre-cooled with liquid nitrogen for fast turn-around and its closed cycled cooler can maintain the inner chamber as cold as $12 \mathrm{~K}$.

We have tested several of the FPM's and have obtained noise numbers for the Orion I readout. These numbers are for shorted input noise and are given in $\mu$ volts as we cannot accurately do a conversion to electrons at this time. With the hybrid system described above, we are getting $35 \mu \mathrm{V}$ RMS for a single read and $40 \mu \mathrm{V}$ RMS with Fowler_1 sampling. It is worth noting that we been able to attain dark current well below 0.1 electrons/sec and read noise below 7 electrons RMS (Fowler_81) with ALADDIN III SCAs.

\subsection{Future Work}

Much work needs to be done on characterizing these devices. Since the Orion detectors are being processed and AR coated the same as the ALADDIN devices, we do not anticipate any surprises in the area of quantum efficiency. We have learned some things on the ALADDIN program that lead us to believe we may have a solution to the image retention problem (should attempts to ameliorate the situation during manufacture fail), but that will have to be proven on future devices. Based on our electrical tests to date, we expect the read noise for Fowler_1 sampling will be in the 20 electrons 
RMS range, but we have not proven that yet. So look for more test data and information on performance of these devices in the future. In addition to characterization of the Orion II multiplexers and the Orion II FPMs, we intend to establish efficient optimal strategies for:

- employing the reference pixels to correct for drift in the output video signal

- capitalizing on the capability for switching power to the output transistors to reduce output amplifier glow

- balancing digital filtering and Fowler sampling of the video signal against frame time to assure scientifically productive operation for both low and high backgrounds

\section{ACKNOWLEDGEMENTS}

We want to recognize the extraordinary efforts of the Astronomy Team at RVS. Their work in going from concept to working hardware on the largest infrared focal plane in production is a great achievement. We also wish to acknowledge the financial support of Sidney Wolff of NOAO, NASA, and USNO without which this program would not have been possible.

\section{REFERENCES}

1. A.M. Fowler, K.M. Merrill, W. Ball, A. Henden, F. Vrba, \& C. McCreight, "Orion: A 1-5 Micron Focal Plane for the $21^{\text {st }}$ Century,,, Workshop on Scientific Detectors in Astronomy held in Waimea, Hawaii on July, 2002, to be published by Kluwer Academic Publishers.

2. Albert M. Fowler, Michael Merrill, William J.Ball, Arne A. Henden,. Frederick J.Vrba, \& Craig R. McCreight, "Orion: the Largest Infrared Hybrid Focal Plane Array in Production,,, Proc. SPIE, Vol. 4841, pp. 853-859, Instrument Design and Performance for Optical/Infrared Ground-based Telescopes, Masanori Iye and Alan F. Moorwood, Eds., 2003.

3. Alan W. Hoffman, Peter J. Love, Ken J. Ando, Joseph P. Rosbeck, Nancy A. Lum, Roger Holcombe, John M. Durkee, \& Craig W. McMurtry, "Development of 2k x 2k FPA InSb modules for the NGST mission,, Proc. SPIE, Vol. 4850, pp. 836-846, IR Space Telescopes and Instruments, John C. Mather, Ed., 2003.

4. Roy G. Autry, Ronald G. Probst, Barry M. Starr, Khairy M. Abdel-Gawad, Rick D. Blakley, Philip N. Daly, Ruben Dominguez, Edward A. Hileman, Ming Liang, Earl T. Pearson, Richard A.Shaw, \& Doug Tody , "NEWFIRM: the widefield IR imager for NOAO 4-m telescopes,,, Proc. SPIE, Vol. 4841, pp. 525-539, Instrument Design and Performance for Optical/Infrared Ground-based Telescopes, Masanori Iye and Alan F. Moorwood, Eds., 2003.

5. Gert Finger, Hamid Mehrgan, Manfred Meyer, Alan F Moorwood,. Gianalfredo Nicolini, \& Joerg Stegmeier, "Performance of large-format $\mathrm{HgCdTe}$ and InSb arrays for low-background applications," Proc. SPIE, Vol. 4008, pp. 1280-1297, Optical and IR Telescope Instrumentation and Detectors, Masanori Iye and Alan F. Moorwood, Eds., 2003.

6. Albert M. Fowler, Ian Gatley, Paul McIntyre, Frederick J Vrba, \& Alan W. Hoffman, "ALADDIN, the 1024x1024 InSb array: design, description, and results,, Proc. SPIE, Vol. 2816, p. 150-160, Infrared Detectors for Remote Sensing: Physics, Materials, and Devices, Randolph E. Longshore and Jan W. Baars, Eds., 1999.

7. Peter J. Love, Ken J.Ando, Richard E. Bornfreund, Elizabeth Corrales, Robert E. Mills, Jerry R. Cripe, Nancy A. Lum, Joseph P. Rosbeck, \& Michael S. Smith, "Large-format infrared arrays for future space and ground-based astronomy applications,,, Proc. SPIE, Vol. 4486, pp. 373-384, Infrared Spaceborne Remote Sensing IX, Marija Strojnik and Bjorn F. Andresen, Eds., 2002.

8. Barry M. Starr, Nick C. Buchholz, Gustavo Rahmer, Gerald Penegor, Ricardo E. Schmidt, Michael Warner, Michael Merrill, Charles F Claver, Y. Ho, K. N. Chopra, C. Shroff, \& D. Shroff, "MONSOON: Image Acquisition System or 'Pixel Server',,, Proc. SPIE, Vol. 4841, pp. 600-611, Instrument Design and Performance for Optical/Infrared Ground-based Telescopes, Masanori Iye and Alan F. Moorwood, Eds., 2003.

9. Gerald B. Heim, Nick C. Buchholz, \& Roger W. Luce, "NOAO Wildfire Instrument Controller,,, Proc. SPIE, Vol. 2198, pp. 1024-1035, Instrumentation in Astronomy VIII, David L. Crawford and Eric R. Craine, Eds., 1994.

10. Gerald B Heim, Nick C. Buchholz, \& Albert M. Fowler, "Controlling an ALADDIN 1Kx1K array with Wildfire,", Proc. SPIE, Vol. 2475, pp. 118-129, Infrared Detectors and Instrumentation for Astronomy, Albert M. Fowler, Ed., 1995.

11. Wendy Harrison, Albert M. Fowler, \& William Ball, "Characterization of Gemini near-IR arrays,,, Proc. SPIE, Vol. 3354, pp. 214-221, Infrared Astronomical Instrumentation, Albert M. Fowler, Ed., 1998. 